\title{
Sacubitril-Valsartan Compared With Enalapril for the Treatment of Heart Failure: A Decision-Analytic Markov Model Simulation in China
}

\author{
Yue Wu ${ }^{1}$, Shuo Tian ${ }^{1,2}$, Peipei Rong ${ }^{1}$, Fan Zhang ${ }^{1}$, Ying Chen ${ }^{1}$, Xianxi Guo ${ }^{1}$ \\ and Benhong Zhou ${ }^{1,2 *}$ \\ ${ }^{1}$ Department of Pharmacy, Renmin Hospital, Wuhan University, Wuhan, China, ${ }^{2}$ School of Pharmaceutical Sciences, Wuhan \\ University, Wuhan, China
}

OPEN ACCESS

Edited by:

Tanveer Ahmed Khan,

National Institute of Health, Pakistan

Reviewed by:

Adina Turcu-Stiolica,

University of Medicine and Pharmacy

of Craiova, Romania

Brian Godman,

Karolinska Institutet (KI), Sweden

${ }^{*}$ Correspondence:

Benhong Zhou

benhongzh@whu.edu.cn

Specialty section:

This article was submitted to

Pharmaceutical Medicine and Outcomes Research,

a section of the journal

Frontiers in Pharmacology

Received: 27 March 2020

Accepted: 06 July 2020

Published: 23 July 2020

Citation:

Wu Y, Tian S, Rong P, Zhang F, Chen Y, Guo X and Zhou B (2020) Sacubitril-Valsartan Compared With Enalapril for the Treatment of Heart Failure: A Decision-Analytic Markov Model Simulation in China.

Front. Pharmacol. 11:1101. doi: 10.3389/fphar.2020.01101
Objectives: Heart failure with reduced ejection fraction (HFrEF) is a major health concern globally due to high mortality rates, frequent hospitalization and considerable medical expenditure. The prevalence of HFrEF is steadily rising in Asian countries, and populous, developing countries like China are facing a significant socio-economic burden as a result. Sacubitril-valsartan (Sac-Val) is currently a class I recommendation for treating HFrEF in major guidelines, although it has not been pharmaco-economically evaluated in China. To this end, we compared the cost-effectiveness of Sac-Val and enalapril based on the negotiated prices in order to fully assess the expected costs and benefits of the clinical use of Sac-Val in China.

Method: A Markov model was constructed to estimate long-term clinical and economic outcomes of Sac-Val versus enalapril for HFrEF patients in China over a 10-year horizon. Primary model outcomes were total costs and quality-adjusted life years (QALYS), and the incremental cost-effectiveness ratio (ICER).

Results: Treatment with Sac-Val resulted in 4.67 QALYs at the cost of $\$ 4,684.25$, while enalapril yielded 4.40 QALYs at the cost of $\$ 4,014.47$. Compared to enalapril, Sac-Val was associated with a gain of 0.27 QALYs, resulting in an ICER of $\$ 2,480.67$ per QALY. Deterministic sensitivity analysis showed robust results. Probabilistic sensitivity analysis suggested that Sac-Val has a 99.99\% probability of being cost-effective at the willingnessto-pay threshold of $\$ 10,276$.

Conclusion: From Chinese patients' perspective, Sac-Val is a cost-effective treatment option for HFrEF in China compared to enalapril. Our findings can aid clinicians plan the Sac-Val regimen, as well as decision makers to discuss the value and position of novel angiotensin receptor neprilysin inhibitors (ARNIs) in future.

Keywords: sacubitril-valsartan, enalapril, cost effectiveness, heart failure, China 


\section{INTRODUCTION}

Heart failure (HF) is a major health concern worldwide due to the elevated mortality rates, frequent hospitalization and high expenditures (Savarese and Lund, 2017). Globally, HF affects an estimated 38 million people, with a $1 \%-2 \%$ prevalence in the adult population (Braunwald, 2015). Depending on extent of the decline in left ventricular ejection function (LVEF), HF is typically classified into heart failure with preserved ejection fraction (HFpEF) and with reduced ejection fraction (HFrEF) types. However, patients with HF have poor prognosis regardless of the LVEF, with a poor survival rate of $<50 \%$ within 5 years after the first hospitalization (Tribouilloy et al., 2008; Buddeke et al., 2020). In addition, almost half of the HF patients suffer from fatigue, breathlessness, edema or arrhythmias, which significantly reduces the quality of life (Gohler et al., 2009). HF is also associated with high rates of hospitalization and readmission (Cook et al., 2014), and accounts for $1 \%-2 \%$ of the overall hospitalizations in USA and Europe (Ambrosy et al., 2014).

The etiology of HF is variable depending on the geographical region but hypertension and coronary artery disease are the major risk factors. Regular physical activity and maintaining a healthy BMI can lower the risk of HF or at least slow disease progression (Horwich and Fonarow, 2017). However, majority of the HF patients require drug therapy, especially those with significant loss of cardiac function. ACE inhibitors (ACEIs) are typically the first-line treatment for HFrEF over the past few decades. Despite that ACEIs reduce the risk of hospitalization and death, the rates of hospitalization and death remain high as well as the loss of quality of life in HF patients (McMurray et al., 2018). The combination of the neprilysin inhibitor sacubitril and angiotensin-II receptor blocker (ARB) valsartan (Sac-Val) was proposed as a promising alternative for treating HFrEF in the last decade. PARADIGM-HF, a multinational phase III, doubleblind, prospective randomized clinical trial (McMurray et al., 2014), showed that Sac-Val was superior to enalapril in terms of reducing the composite primary outcome of cardiovascular (CV) death or hospitalization in HFrEF patients. Based on their findings, Sac-Val is now a class I recommendation in the recent guidelines for the management of HF in USA (Yancy et al., 2017), Europe (Ponikowski et al., 2016) and China (Chinese Medical Association et al., 2019) alongside other standard therapies.

Apart from the therapeutic effects, the results from PARADIGM-HF have also been used for evaluating the economic viability of Sac-Val (Liu et al., 2020), especially in the developed countries (Gaziano et al., 2016; King et al., 2016; Sandhu et al., 2016; van der Pol et al., 2017; Zanfina et al., 2017; Gandjour and Ostwald, 2018; Krittayaphong and Permsuwan, 2018; Liang et al., 2018; Zueger et al., 2018; Park et al., 2019; van der Pol et al., 2019). Owing to the high willingness-to-pay (WTP) threshold, Sac-Val is likely to be cost-effective compared to enalapril in United States (Gaziano et al., 2016; King et al., 2016; Sandhu et al., 2016), United Kingdom (McMurray et al.,
2018) and some other European countries (Zanfina et al., 2017; Gandjour and Ostwald, 2018; McMurray et al., 2018; van der Pol et al., 2019) despite the higher acquisition cost. However, few studies have evaluated the pharmaco-economics of Sac-Val in the low and middle-income Asian countries.

China is the largest developing country in Asia with a population of 1.3 billion, and is now facing a heavier socioeconomic burden of HF compared to the West. With a steadily aging population, and increasing incidence of cardiovascular risk factors like obesity, hypertension, and diabetes, the prevalence of HF has significantly risen in China in recent years (Huang et al., 2017; Zhang et al., 2017). Due to the large population and limited economic resources, China has more HF patients with lower capacity for bearing the costs of medical treatment. Furthermore, the clinical phenotypes and treatment patterns also vary considerably between the developed and developing countries, which usually translates to similarly poor or even worse outcomes in Asian population (Mentz et al., 2016). Given that pharmaco- economic evaluation is greatly affected by the economic capacity and patient subgroups, the expected costs and benefits of Sac-Val treatment in China remain unknown.

In 2017, in order to further improved the quality of medical care, the Chinese government launched the national negotiation on the prices of groups of drugs covered by medical insurance. Sac-Val was listed in the negotiating drug catalog in 2019, and is covered by the national insurance reimbursement, which could greatly increase its affordability. In this study, we compared the cost-effectiveness of Sac-Val and enalapril based on the current price control mechanism in the Asian subgroup, in order to fully assess the expected costs and benefits of using Sac-Val in China.

\section{METHODS}

\section{Model Structure}

A decision-analysis state-transition Markov model with onemonth cycles and 10-year horizon was created to compare SacVal versus enalapril for HFrEF patients in China (Figure 1). Five Markov states-including the New York Heart Association (NYHA) classes I to IV and deaths-were defined in the model. Within-cycle transitions state included HF hospitalization, 30-day readmission, progression to subsequent NYHA class, cardiovascular (CV) death and non-CV death. The assumption was made that during each cycle, patients may either remain in their current health state or transition to the next state due to an event. The starting age of the patient cohort was set at 64 years (Liu et al., 2014) in accordance with the mean age of Chinese population with HF. The probability of beginning the first cycle in a given NYHA class was determined according to the characteristic of PARADIGM-HF at the time of randomization (4.5\% NYHA I, 71.6\% NYHA II, 23.1\% NYHA III, 0.8\% NYHA IV) (McMurray et al., 2014). A 10-year time horizon was used based on average life expectancy of 5 to 10 years for HF patients (Allen et al., 2008; Alter et al., 2012). Model 


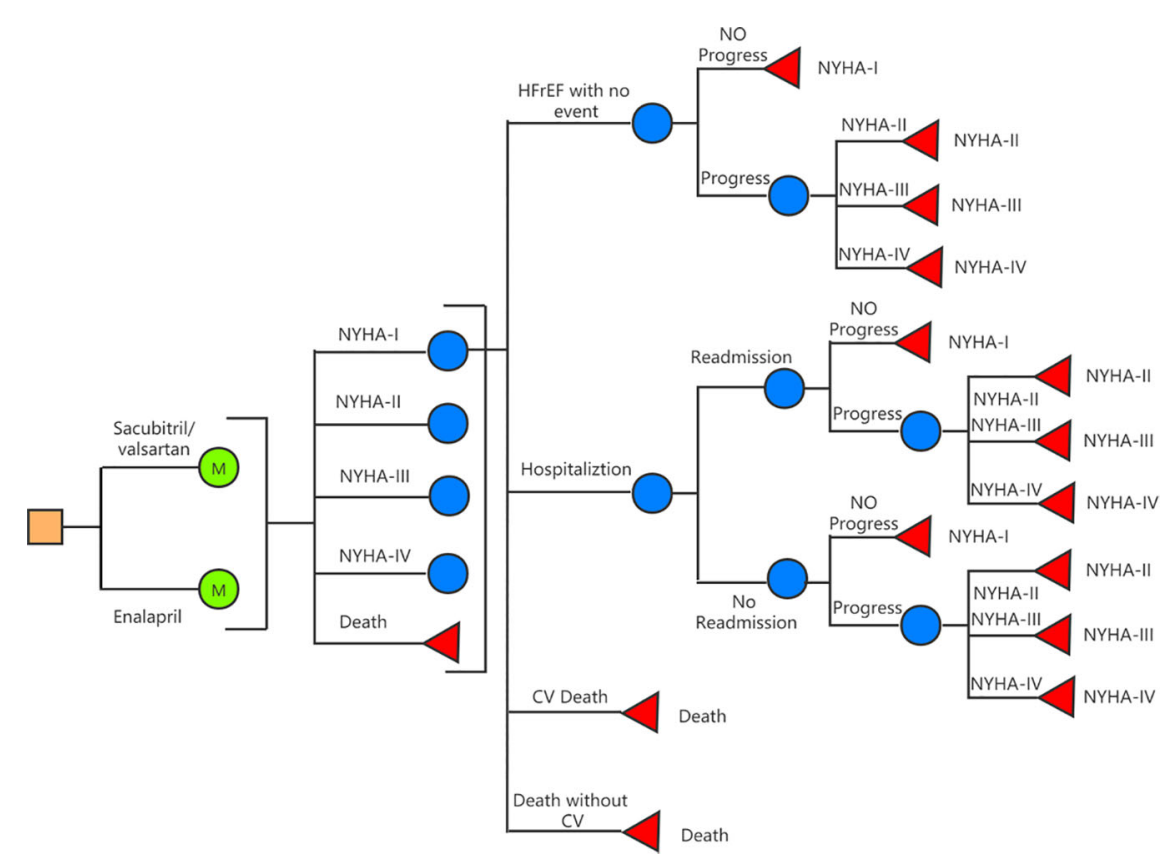

FIGURE 1 | Schematic representation of the Markov model.

construction and analyses were performed using TreeAge Pro SuiteTM software 2019 (Williamstown, MA. USA).

\section{Date and Sources Probability}

A targeted literature review was conducted to identify appropriate model inputs (Table 1), and the model was developed in a hypothetical cohort based on the characteristics observed in PARADIGM-HF trial. The baseline probabilities of hospitalization and CV death were derived from the KaplanMeier curves of enalapril group. Weibull and Exponential distribution were fitted to obtain the individual monthly transition probabilities (Liang et al., 2018). The clinical benefits of Sac-Val were modeled by applying the hazard ratios (HRs) to the baseline probabilities, and the NYHA class-specific probabilities for hospitalization and CV deaths were obtained by applying the hazard ratios (HRs) and relative risks (RRs) to the baseline probabilities. Age-dependent non-CV mortality was derived from local epidemiological data (2018) after excluding CV deaths (Table 2). The readmission rate of $16.23 \%$ was derived from the national insurance database including 7,847 patients with HF (Huang et al., 2017). Readmission rates were assumed to be the same in both arms because no significant difference was observed in Asia-Pacific subgroup of PARADIGM-HF (Desai et al., 2016). NYHA progressing probabilities in Sac-Val and enalapril treatment arms were 0.0068 and 0.0088 as reported previously (Zueger et al., 2018). NYHA class specific transition probabilities were cited from an established matrix (King et al., 2016) (Table 3), and were assumed to be fixed in one-month cycle and same in both treatment arms, since the effect of Sac-Val on NYHA class transition relative to that of enalapril is unclear.

\section{Cost and Utilities}

Health utility values described the quality of life for each health states were obtained from published studies (Table 1) and were scored according to a scale ranging from 0 (death) to 1 (perfect health). One-time dis-utilities of -0.1 was used for each hospitalization and readmission event in NYHA I-IV (King et al., 2016). Consistent with the perspective of the Chinese patients, the model only incorporated the direct healthcare costs of HF therapy, and a half-cycle correction was applied in the model. Drug costs were derived from the prices charged for $10 \mathrm{mg}$ enalapril twice daily and $200 \mathrm{mg}$ Sac-Val twice daily (Table 4) in China. Considering the differences in drug quality between foreign and local manufacturers, the generic price of enalapril produced by overseas pharmaceutical companies was used in base case analysis. The costs for HF-associated hospitalization, readmission and doctor visits were derived from local epidemiological data (Huang et al., 2017), and assumed to be equivalent across both treatment groups. All the costs inputs were inflated to 2019 . Since that the discount rate is not explicitly recommended in China, the usual discount rate of 3.5\% per year was used to eliminate the effects of inflation.

\section{Outcomes}

The main outcome measured in this analysis was incremental costs per quality-adjusted life-year (QALY) gained, expressed as the incremental cost-effectiveness ratio (ICER). Since there is no fixed 
TABLE 1 | Parameters inputs.

\begin{tabular}{|c|c|c|c|c|c|c|}
\hline Input variable & Vaule & Low & Up & Distribution & Source & Notes \\
\hline \multicolumn{7}{|l|}{ Baseline Probability } \\
\hline $\begin{array}{l}\text { Weibull model for hospitalization } \\
\text { in enalapril }\end{array}$ & $\begin{array}{l}\lambda=-0.00097 \\
\gamma=1.02685\end{array}$ & 0.00868 & 0.0145 & - & $\begin{array}{l}\text { (McMurray et al., 2014; King et al., } \\
\text { 2016) }\end{array}$ & $\begin{array}{l}\text { Extrapolated from published } \\
\text { Kaplan-Meier curves from }\end{array}$ \\
\hline $\begin{array}{l}\text { Exponential model for CV death in } \\
\text { enalapril }\end{array}$ & $\lambda=-0.00577$ & 0.00412 & 0.00844 & - & $\begin{array}{l}\text { (McMurray et al., 2014; King et al., } \\
\text { 2016) }\end{array}$ & PARADIGM-HF trial \\
\hline Readmission & 0.0147 & 0.0132 & 0.0161 & Beta & (Desai et al., 2016) & $\pm 10 \%$ of the mean \\
\hline NYHA Progress for enalapril & 0.0088 & 0.0079 & 0.0097 & Beta & (Zueger et al., 2018) & $\pm 10 \%$ of the mean \\
\hline NYHA Progress for Sac-val & 0.0068 & 0.00612 & 0.00748 & Beta & (Zueger et al., 2018) & $\pm 10 \%$ of the mean \\
\hline Background no-CV mortality & \multicolumn{4}{|c|}{ Aged specific } & $\begin{array}{l}\text { (National Health Commission of the } \\
\text { People's Republic of China, 2018) }\end{array}$ & - \\
\hline \multicolumn{7}{|c|}{ Hazard ratios (HR)/Risk ratio(RR) compared to baseline* } \\
\hline HR of Hospitalization for Sac-val & 0.79 & 0.71 & 0.89 & Log-normal & (McMurray et al., 2014) & $95 \% \mathrm{Cl}$ \\
\hline HR of CV death for Sac-val & 0.80 & 0.71 & 0.89 & Log-normal & (McMurray et al., 2014) & $95 \% \mathrm{Cl}$ \\
\hline HR of Hospitalization for NYHA III & 1.71 & 1.33 & 2.18 & Log-normal & (Ahmed et al., 2006) & $95 \% \mathrm{Cl}$ \\
\hline HR of Hospitalization for NYHA IV & 3.4 & 1.69 & 6.84 & Log-normal & (Ahmed et al., 2006) & $95 \% \mathrm{Cl}$ \\
\hline $\begin{array}{l}\text { RR of CV death for NYHA III to } \\
\text { baseline }\end{array}$ & 1.372 & 1.303 & 1.445 & Log-normal & (Pocock et al., 2012) & $95 \% \mathrm{Cl}$ \\
\hline RR of CV death for NYHA IV & 1.640 & 1.503 & 1.790 & Log-normal & (Pocock et al., 2012) & $95 \% \mathrm{Cl}$ \\
\hline \multicolumn{7}{|l|}{ Utility inputs } \\
\hline NYHA I and II & 0.780 & 0.741 & 0.819 & Beta & (Xuan et al., 2017) & $\pm 5 \%$ of the mean; \\
\hline NYHA III & 0.715 & 0.679 & 0.751 & Beta & (Xuan et al., 2017) & $\pm 5 \%$ of the mean; \\
\hline NYHA IV & 0.660 & 0.627 & 0.693 & Beta & (Xuan et al., 2017) & $\pm 5 \%$ of the mean; \\
\hline \multicolumn{7}{|l|}{ Disutility (one-time) } \\
\hline Hospitalization & -0.1 & -0.13 & -0.08 & Beta & (King et al., 2016) & $95 \% \mathrm{Cl}$ \\
\hline Readmission & -0.1 & -0.13 & -0.08 & Beta & (King et al., 2016) & $95 \% \mathrm{Cl}$ \\
\hline \multicolumn{7}{|l|}{ Cost inputs } \\
\hline Sac-Val & 17.12 & 13.70 & 30.46 & Gamma & Local date & Local date \\
\hline Enalapril & 10.65 & 0.90 & 12.78 & Gamma & Local date & Local date \\
\hline Outpatient visit & 41.56 & 33.26 & 49.87 & Gamma & Local date & $\pm 20 \%$ of the mean; \\
\hline Copay ratio for inpatient & 0.3 & 0.1 & 1 & - & Local date & - \\
\hline \multicolumn{7}{|l|}{ Cost of events (one-time) } \\
\hline Hospitalization & $1,920.49$ & $1,536.39$ & $2,304.59$ & Gamma & (Huang et al., 2017) & $\pm 20 \%$ of the mean; \\
\hline Readmission & $1,340.05$ & $1,072.04$ & $1,608.06$ & Gamma & (Huang et al., 2017) & $\pm 20 \%$ of the mean; \\
\hline
\end{tabular}

*The baseline probabilities were assumed to the probabilities of NYHA class I and II.

TABLE 2 | Background no-cardiovascular (CV) mortality.

\begin{tabular}{cccc}
\hline Age & Background no-CV mortality & Age & Background no-CV mortality \\
\hline 1 & 0.002949 & 50 & 0.0011998 \\
5 & 0.000284 & 55 & 0.0029394 \\
10 & 0.00015 & 60 & 0.0028332 \\
15 & 0.000169 & 65 & 0.0066972 \\
20 & 0.000177 & 70 & 0.0097194 \\
25 & 0.000154 & 75 & 0.0121676 \\
30 & 0.000298 & 80 & 0.016739 \\
35 & 0.000458 & 85 & 0.0331037 \\
40 & 0.000472 & 100 & 1.00 \\
45 & 0.00071 & &
\end{tabular}

TABLE 3 | New York Heart Association (NYHA) progressing probabilities per one-month cycle.

\begin{tabular}{lcccc}
\hline \multirow{2}{*}{ From NYHA } & \multicolumn{4}{c}{ To HYHA } \\
\cline { 2 - 5 } & Class I & Class II & ClassIII & Class IV \\
\hline Class I & - & 0.831 & 0.169 & 0 \\
Class II & 0.422 & - & 0.531 & 0.047 \\
Class III & 0 & 0.847 & - & 0.153 \\
Class IV & 0 & 0 & 1.000 & -
\end{tabular}

WTP threshold currently to determine cost-effectiveness in China, the gross domestic product (GDP) per capita $(\$ 10,276)$ and threefold GDP per capital $(\$ 30,828)$ were used as the threshold. Cost effectiveness was assessed annually to determine at which point in time the treatment options achieved acceptable levels.

\section{Sensitivity Analysis}

One-way, two-way and probabilistic sensitivity analysis (PSA) were performed to assess the impact of parameter uncertainty on the results. Model parameters were varied over their 95\% confidence intervals. Variations of $\pm 5 \%, \pm 10 \%$, and $\pm 20 \%$ were assumed for parameters of utility, probability and medical costs that have no specified data range. The range of the cost for Sac-Val and enalapril were set based on the distribution of local prices (including all products from foreign and local manufacturers). In PSA, probabilities of clinical outcomes and health utilities were assigned as beta distributions. Log-normal distributions were applied for HRs and RRs. Gamma distributions were assumed for drug and healthcare costs. All variables were allowed to simultaneously vary stochastically in PSA. A second-order Monte Carlo simulation was performed $(n=10,000)$ based on the variablespecific distributions. Results of the PSA were presented graphically as scatterplots and cost-effectiveness acceptability curves. 
TABLE 4 | Drug dose and costs.

\begin{tabular}{|c|c|c|c|c|c|}
\hline Drug & Dose & Unit Price (\$) & Medicare copay ratio* & Monthly price(\$) & Monthly cost(\$) \\
\hline Sac-val & 200 mg twice daily & $1.42 / 200 \mathrm{mg}$ & 0.8 & 85.61 & 17.12 \\
\hline Enalapril & 10 mg twice daily & $0.18 / 10 \mathrm{mg}$ & 0 & 10.65 & 10.65 \\
\hline
\end{tabular}

${ }^{*}$ Medicare copay ratio for outpatient.

\section{RESULTS}

\section{Base-Case Analysis}

Over a 10 -year horizon, $51.3 \%$ patients were predicted to die in the Sac-Val cohort compared with $58.1 \%$ in the enalapril cohort. The predicted costs, benefits and ICERs of the two arms are summarized in Table 5. Furthermore, treatment with Sac-Val was predicted to yield 4.67 QALYs at the cost of $\$ 4,684.25$, while enalapril yielded 4.40 QALYs at the cost of $\$ 4,014.47$. This resulted in an ICER of $\$ 2,480.67$ per QALY gained for Sac-Val versus enalapril, which is much lower than the costeffectiveness threshold.

Varying the time horizon significantly affected the costeffectiveness estimates (Table 5). At 5 years of follow-up, the ICER per QALY gained was $\$ 3,684.57$, which decreased to $\$ 2,032.52$ and $\$ 1,874.00$ when the time horizon extended to 15 and 20 years respectively. The effect of the initial NYHA class distribution was also calculated, and showed that a higher proportion of NYHA class III and IV patients, in accordance with epidemiological characteristics of HF in China, increased the estimated ICER to $\$ 2,697.82$ per QALY gained.

\section{Sensitivity Analysis One-Way Sensitive Analysis}

A tornado analysis was performed to evaluate the impact of input parameters (Figure 2). The prices of Sac-Val and enalapril had the largest impact on ICER, followed by the hazard risk of CV deaths, CV mortality and the cost of outpatient visit. At the highest end of Sac-Val cost or the lowest end of enalapril cost, ICER respectively increased to $\$ 5,992.46$ or $\$ 4,878.37$ per QALY. Other input parameters, such as health utilities, therapeutic costs and risks for clinical events, had a minor impact on estimated
ICER. Furthermore, the ICER values did not increase over the cost-effectiveness threshold when varying any model inputs across their plausible ranges, indicating that Sac-Val is robustly cost-effective compared to enalapril.

\section{Two-Way Sensitive Analysis}

To fully assessed the potential impact of price negotiation on the outcomes, two-way sensitive and threshold analysis was conducted by extending the cost range of Sac-Val to $\$ 150$ percycle, which is $\sim 500 \%$ of the highest end of the cost (Figure 3). Based on the current cost of enalapril at $\$ 0.9$ to $\$ 12.78$ permonth, Sac-Val will have to cost $\$ 37.25-\$ 48.44$ per-month to be considered cost-effective with the WTP threshold of $\$ 10,276$. When threefold GDP per capital $(\$ 30,828)$ was applied as the WTP threshold as recommended by World Health Organization (WHO), the monthly cost of Sac-val could increase to \$113.91-\$125.10.

\section{Probabilistic Sensitivity Analysis}

As shown in the PSA simulation scatterplot in Figure 4, Sac-Val had a higher average total cost of \$ 4,522.11 (95\% CI, \$3,341.23$\$ 5,905.97$ ) compared to $\$ 3,846.04$ (95\% CI, \$2,808.77-\$ 5083.80) of enalapril. Over a 10-year horizon, Sac-Val gained average QALYs of 4.21 (95\% CI, 4.18-4.23) compared to 4.51 (95\% CI, 4.49-4.54) gained by enalapril. A cost-effectiveness acceptability curve was plotted to demonstrate the proportion of simulations that were cost-effective at the WTP values (Figure 5). Compared to enalapril, there was a $99.99 \%$ probability that Sac-Val is cost-effective using a WTP threshold of $\$ 10,276$. The cost-effectiveness probabilities did not change significantly when the monthly costs of Sac-Val varied between $80 \%$ and $120 \%$. If the costs further increased to $\$ 113.91$ and $\$ 125.10$, the

TABLE 5 | Base case analysis and one-way sensitivity analysis on time horizon and initial New York Heart Association (NYHA) class distribution.

\begin{tabular}{|c|c|c|c|c|c|c|}
\hline & Treatment strategies & Cost(\$) & Incremental cost (\$) & QALY & Incremental QALYs & ICER(\$/QALYs) \\
\hline Base case & $\begin{array}{l}\text { Sac-val } \\
\text { Enalapril }\end{array}$ & $\begin{array}{l}4,684.25 \\
4,014.47\end{array}$ & 669.78 & $\begin{array}{l}4.67 \\
4.40\end{array}$ & 0.27 & $2,480.67$ \\
\hline \multicolumn{7}{|c|}{ Time horizon } \\
\hline 5 -years & $\begin{array}{l}\text { Sac-val } \\
\text { Enalapril }\end{array}$ & $\begin{array}{l}2,973.81 \\
2,610.82\end{array}$ & 362.99 & $\begin{array}{l}2.95 \\
2.85\end{array}$ & 0.10 & $3,684.57$ \\
\hline 15-years & $\begin{array}{l}\text { Sac-val } \\
\text { Enalapril }\end{array}$ & $\begin{array}{l}5,661.36 \\
4,760.89\end{array}$ & 900.47 & $\begin{array}{l}5.64 \\
5.20\end{array}$ & 0.44 & $2,032.52$ \\
\hline 20-years & $\begin{array}{l}\text { Sac-val } \\
\text { Enalapril }\end{array}$ & $\begin{array}{c}6,170.73 \\
5,121.5\end{array}$ & $1,049.23$ & $\begin{array}{l}6.14 \\
5.58\end{array}$ & 0.56 & $1,874.00$ \\
\hline \multicolumn{7}{|c|}{ Initial NYHA class distribution:5\% I; 20\% II; 45\% III; 30\% IV } \\
\hline 5 -years & $\begin{array}{l}\text { Sac-val } \\
\text { Enalapril }\end{array}$ & $\begin{array}{l}2,995.24 \\
2,615.57\end{array}$ & 379.67 & $\begin{array}{l}2.73 \\
2.64\end{array}$ & 0.09 & $4,175.26$ \\
\hline 10-years & $\begin{array}{l}\text { Sac-val } \\
\text { Enalapril }\end{array}$ & $\begin{array}{l}4,609.59 \\
3,921.93\end{array}$ & 687.66 & $\begin{array}{l}4.33 \\
4.08\end{array}$ & 0.25 & $2,697.82$ \\
\hline 20-years & $\begin{array}{l}\text { Sac-val } \\
\text { Enalapril }\end{array}$ & $\begin{array}{l}5,977.42 \\
4,933.07\end{array}$ & $1,044.35$ & $\begin{array}{l}5.69 \\
5.17\end{array}$ & 0.51 & $2,030.94$ \\
\hline
\end{tabular}




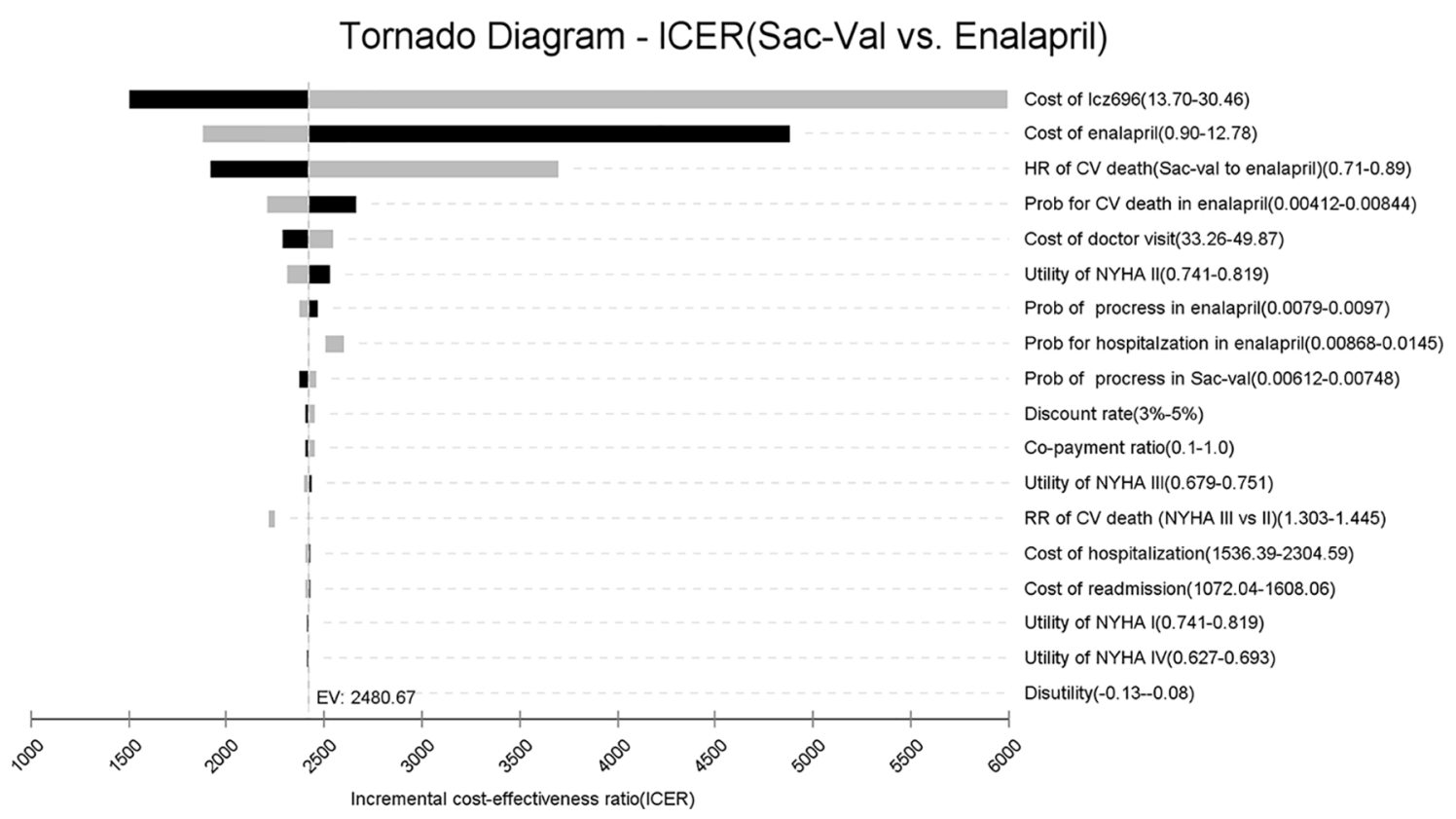

FIGURE 2 | Tornado analysis: ICER of Sac-val vs. enalapril over plausible ranges of model inputs.

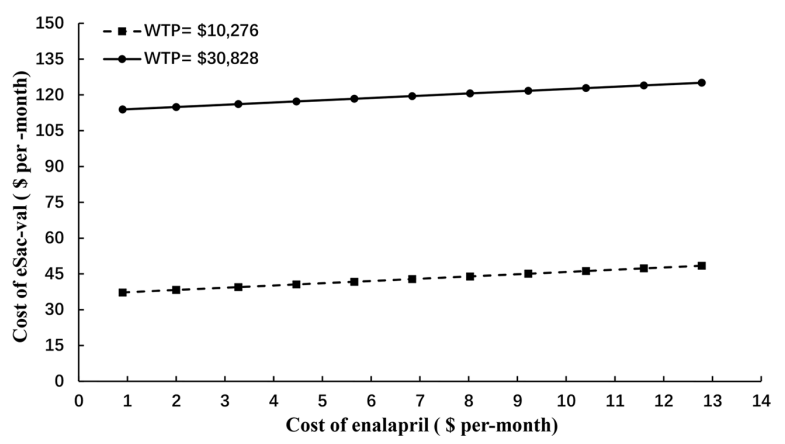

FIGURE 3 | Two-way sensitive analysis on the cost of Sac-val and enalapril.

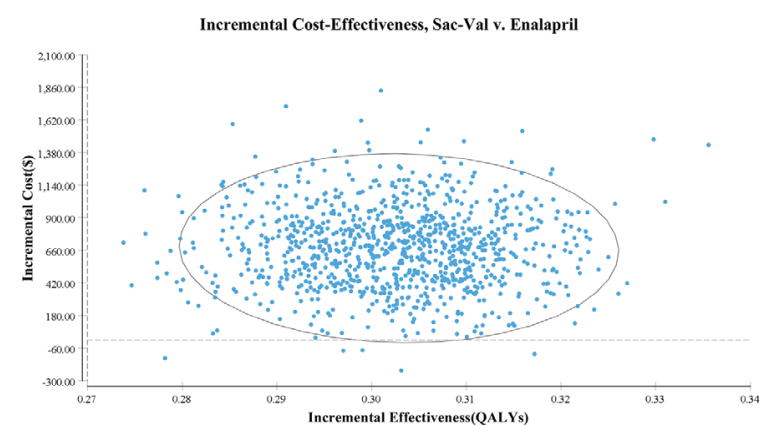

FIGURE 4 | Scatter plot of the probabilistic sensitivity analyses.

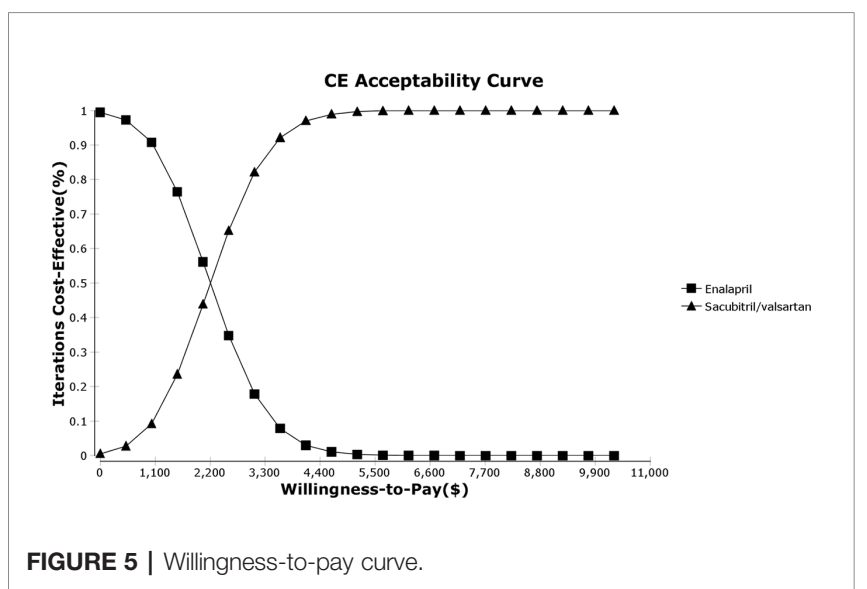

probability of cost-effectiveness ranged from $86.2 \%$ to $69.7 \%$ at a WTP of $\$ 30,828 /$ QALY gained.

\section{DISCUSSION}

The prevalence of HFrEF is steadily increasing in Asian countries, resulting in considerable socioeconomic burden due to huge population and limited economic resources. The novel combination drug Sac-Val has yielded clinical benefits in HFrEF patients. Given the low-cost generic status of ACEIs, Sac-Val should be pharmaco-economically evaluated in Asia to determine whether its clinical benefits are worth the additional 
costs. This is the first study to compare the cost-effectiveness of Sac-Val and enalapril in China based on the health costs and resources, which can further enable informed healthcare decision-making in developing countries.

The Chinese government launched national price negotiation between the National Health Insurance Administration and drug producers in 2017 in order to further improve the quality of medical care. Novel drugs with significant clinical efficacy but high costs can only be included in the medical insurance reimbursement list after the price is reduced. The reimbursement list was renewed on Jan 1, 2020, and included several drugs that can improve patient quality of life, such as SacVal. We used the latest negotiated price of Sac-Val to assess its expected costs and benefits in China. Base case analysis showed that Sac-Val is more costly and also more effective compared to enalapril, which is consistent with other cost-effectiveness analyses. In addition, the estimated ICER was significantly lower than the GDP per capital of China regardless of the time horizon, owing to the low negotiated price and insurance coverage. Deterministic sensitivity analysis showed that the results were robust, and probabilistic sensitivity analysis further suggested a $99.99 \%$ probability of Sac-Val being cost-effective

Several studies have compared the cost-effectiveness of SacVal and enalapril in developed countries. Owing to the high WTP threshold, Sac-Val is cost-effective in countries like United States (Gaziano et al., 2016; King et al., 2016; Sandhu et al., 2016), Netherlands (van der Pol et al., 2017), United Kingdom (McMurray et al., 2018) and other European countries (Zanfina et al., 2017; Gandjour and Ostwald, 2018; McMurray et al., 2018; van der Pol et al., 2019), even with high estimated ICERs. In contrast, opposite conclusions were drawn in similar studies conducted in Singapore (Liang et al., 2018) and Thailand (Krittayaphong and Permsuwan, 2018). With a 225-fold higher cost to that of enalapril, Sac-Val was not cost-effective compared to ACEIs in Thailand. Due to the relatively lower WTP (between SGD 20,000 to SGD 50,000) in Singapore, the price of Sac-Val has to be reduced by $32 \%$ to $70 \%$ for it to be cost-effective. Recently, a cost-effect evaluation of Sac-Val in South Korea (Park et al., 2019) concluded that Sac-Val is cost-effective compared to ACEIs, although the ICER was higher than that in our study.

The actual cost of a drug is a key factor in cost-effectiveness analysis. Since the fixed period for the negotiated price of Sac-Val in China is two years (01/2020-12/2021), a two-way sensitive analysis was further conducted by extending its cost range to $\$ 150$ per-month, which simulated the conditions after the contract period. Given the current costs of enalapril at \$0.9$\$ 12.78$ per-month, Sac-Val will have to cost $\$ 37.25-\$ 48.44$ or \$113.91-\$125.10 per-month to be considered cost-effective with WTP threshold of $\$ 10,276$ or $\$ 30,828$ respectively. Based on these results, we recommend that the price of ARNIs should be controlled after the negotiation period as well to increase its affordability. Furthermore, the price increment of Sac-Val should be kept less that sevenfold from the current cost of $\$ 17.12$ per day, in order to be cost-effective over ACEIs in HF treatment.
According to the PARADIGM trial, Sac-Val is superior to enalapril in terms of reducing the risks of death and hospitalization of HF. In this analysis, the ICER displayed sensitivity to the risk and probability of $\mathrm{CV}$ death but was barely affected by the risk of hospitalization. This is consistent with some cost-effectiveness analyses of Sac-Val reported previously (King et al., 2016; Liang et al., 2018; Zueger et al., 2018). Given that HF is a chronic disease with significant mortality, especially in the elderly patients, the modeled population would decline significantly over the projected period due to increasing deaths. As a result, the benefit of SacVal in averting hospitalization might diminish. Furthermore, the lower costs of hospitalization and readmission in China compared to that in some developed countries might further offset the effect of the risk of hospitalization.

The majority of the patients included in the PARADIGM-HF were of NYHA class II with mild symptoms. Although the treatment-related inputs used in the model were obtained from the PARADIGM-HF population, we performed a base-case analysis to assess the cost-effectiveness in the high-risk population. When the proportion of NYHA class III and IV patients were respectively increased to $45 \%$ and $30 \%$ in the model, in order to simulate the epidemiological characteristics of HF in China, the ICERs increased over the 5, 10, and 20-year horizon. According to the results of PARADIGM-HF, the effect size for Sac-Val relative to enalapril varied depending on the baseline risk of CV death or HF-related hospitalization (Simpson et al., 2015). Due to the potentially greater benefit in higher-risk patients, Sac-Val was predicted to be more cost-effective among patients with a higher baseline risk (King et al., 2016). However, we observed a significant decrease in QALYs with a slight reduction in total costs, which eventually increased the ICERs in the high-risk population. This is consistent with a previous report and may attribute to the significant loss of quality of life in NYHA III and IV HF patients. Given the lower ICERs in the PARADIGM-HF population, we recommend the widespread use of $\mathrm{Sac}-\mathrm{Val}$ in the relatively low risk population.

There are several limitations in our study that ought to be addressed. First, the treatment benefits of Sac-Val in this model were derived directly from the PARADIGM-HF trial. Although well designed clinical trials provide robust evidence, the benefits might still be overestimated compared to that in real-world practice. Second, the proportion of high-risk patients is higher in China compared to that in the PARADIGM-HF cohort, which may influence our results if real-world evidence is included. Third, although Asia-Pacific cohort was included in PARADIGM-HF, the raw data in Asian subgroup was absent, which can result in systematic bias and limit the validity of the results. Fourth, due to the lack of long-term follow-up data, the trends observed in PARADIGM-HF, such as improved CV mortality and reduced HF-related hospitalization, were extrapolated beyond the end of the trial period. This is a common limitation shared by most economic evaluation studies. Furthermore, the adverse events and related discontinuation of drug administration were not considered in this study. Sac-Val was associated with higher 
rates of hypotension and non-serious angioedema. Although adverse events reported in PARADIGM-HF trial were rarely required special treatment, the results from our analysis may be conservative.

\section{DATA AVAILABILITY STATEMENT}

The raw data supporting the conclusions of this article will be made available by the authors, without undue reservation, to any qualified researcher.

\section{AUTHOR CONTRIBUTIONS}

YW developed the study protocol, coordinated data collection, performed data analyses, reported study results and drafted the manuscript. ST collected data and revised the manuscript. PR, FZ, YC, and XG developed the study protocol. BZ supervised the work. All authors contributed to the article and approved the submitted version.

\section{REFERENCES}

Ahmed, A., Aronow, W. S., and Fleg, J. L. (2006). Higher New York Heart Association classes and increased mortality and hospitalization in patients with heart failure and preserved left ventricular function. Am. Heart J. 151 (2), 444450. doi: 10.1016/j.ahj.2005.03.066

Allen, L. A., Yager, J. E., Funk, M. J., Levy, W. C., Tulsky, J. A., Bowers, M. T., et al. (2008). Discordance Between Patient-Predicted and Model-Predicted Life Expectancy Among Ambulatory Patients With Heart Failure. JAMA 299 (21), 2533-2542. doi: 10.1001/jama.299.21.2533

Alter, D. A., Ko, D. T., Tu, J. V., Stukel, T. A., Lee, D. S., Laupacis, A., et al. (2012). The Average Lifespan of Patients Discharged from Hospital with Heart Failure. J. Gen. Internal Med. 27 (9), 1171-1179. doi: 10.1007/s11606-012-2072-y

Ambrosy, A. P., Fonarow, G. C., Butler, J., Chioncel, O., Greene, S. J., Vaduganathan, M., et al. (2014). The Global Health and Economic Burden of Hospitalizations for Heart Failure Lessons Learned From Hospitalized Heart Failure Registries. J. Am. Coll. Cardiol. 63 (12), 1123-1133. doi: 10.1016/ j.jacc.2013.11.053

Braunwald, E. (2015). The war against heart failure: the Lancet lecture. Lancet 385 (9970), 812-824. doi: 10.1016/s0140-6736(14)61889-4

Buddeke, J., Valstar, G. B., van Dis, I., Visseren, F. L. J., Rutten, F. H., den Ruijter, H. M., et al. (2020). Mortality after hospital admission for heart failure: improvement over time, equally strong in women as in men. BMC Public Health 20 (1), 36. doi: 10.1186/s12889-019-7934-3

Chinese Medical Association, Chinese Medical Journals Publishing House, Chinese Society of General Practice, Editorial Board of Chinese Journal of General Practitioners of Chinese Medical Association and Expert Group of Guidelines for Primary Care of Cardiovascular Disease (2019). Guideline for primary care of chronic heart failure(2019). Chin. J. Gen. Pract. 18 (10), 936-947. doi: 10.3760/ cma.j.issn.1671-7368.2019.10.008

Cook, C., Cole, G., Asaria, P., Jabbour, R., and Francis, D. P. (2014). The annual global economic burden of heart failure. Int. J. Cardiol. 171 (3), 368-376. doi: 10.1016/j.ijcard.2013.12.028

Desai, A. S., Claggett, B. L., Packer, M., Zile, M. R., Rouleau, J. L., Swedberg, K., et al. (2016). Influence of Sacubitril/Valsartan (LCZ696) on 30-Day Readmission After Heart Failure Hospitalization. J. Am. Coll. Cardiol. 68 (3), 241-248. doi: 10.1016/j.jacc.2016.04.047

Gandjour, A., and Ostwald, D. A. (2018). Sacubitril/Valsartan (LCZ696): A Novel Treatment for Heart Failure and its Estimated Cost Effectiveness, Budget Impact, and Disease Burden Reduction in Germany. Pharmacoeconomics 36 (10), 1285-1296. doi: 10.1007/s40273-018-0688-4

Gaziano, T. A., Fonarow, G. C., Claggett, B., Chan, W. W., Deschaseaux-Voinet, C., Turner, S. J., et al. (2016). Cost-effectiveness Analysis of Sacubitril/ Valsartan vs Enalapril in Patients With Heart Failure and Reduced Ejection Fraction. JAMA Cardiol. 1 (6), 666-672. doi: 10.1001/jamacardio.2016.1747

Gohler, A., Geisler, B. P., Manne, J. M., Kosiborod, M., Zhang, Z. F., Weintraub, W. S., et al. (2009). Utility Estimates for Decision-Analytic Modeling in Chronic Heart Failure-Health States Based on New York Heart Association Classes and Number of Rehospitalizations. Value Health 12 (1), 185-187. doi: 10.1111/j.1524-4733.2008.00425.x

Horwich, T. B., and Fonarow, G. C. (2017). Prevention of Heart Failure. JAMA Cardiol. 2 (1), 116-116. doi: 10.1001/jamacardio.2016.3394

Huang, J., Yin, H., Zhang, M., Ni, Q., and Xuan, J. (2017). Understanding the economic burden of heart failure in China: impact on disease management and resource utilization. J. Med. Econ. 20 (5), 549-553. doi: 10.1080/ 13696998.2017.1297309

King, J. B., Shah, R. U., Bress, A. P., Nelson, R. E., and Bellows, B. K. (2016). CostEffectiveness of Sacubitril-Valsartan Combination Therapy Compared With Enalapril for the Treatment of Heart Failure With Reduced Ejection Fraction. JACC: Heart Failure 4 (5), 392-402. doi: 10.1016/j.jchf.2016.02.007

Krittayaphong, R., and Permsuwan, U. (2018). Cost-Effectiveness Analysis of Sacubitril-Valsartan Compared with Enalapril in Patients with Heart Failure with Reduced Ejection Fraction in Thailand. Am. J. Cardiovasc. Drugs 18 (5), 405-413. doi: 10.1007/s40256-018-0288-x

Liang, L., Wu, D. B., Aziz, M. I. A., Wong, R., Sim, D., Leong, K. T. G., et al. (2018). Cost-effectiveness of sacubitril/valsartan versus enalapril in patients with heart failure and reduced ejection fraction. J. Med. Econ. 21 (2), 174-181. doi: 10.1080/13696998.2017.1387119

Liu, X., Yu, H., Pei, J., Chu, J., Pu, J., and Zhang, S. (2014). Clinical Characteristics and Long-term Prognosis in Patients with Chronic Heart Failure and Reduced Ejection Fraction in China. Heart Lung Circ. 23 (9), 818-826. doi: 10.1016/ j.hlc.2014.02.022

Liu, X. Q., He, L. S., Huang, J. Q., Xiong, L. J., Xia, C., and Lao, H. Y. (2020). Costeffectiveness analyses of sacubitril-valsartan for heart failure. Heart Fail Rev. doi: 10.1007/s10741-020-09956-6

McMurray, J. J. V., Packer, M., Desai, A. S., Gong, J., Lefkowitz, M. P., Rizkala, A. R., et al. (2014). Angiotensin-Neprilysin Inhibition versus Enalapril in Heart Failure. N Engl. J. Med. 371 (11), 993-1004. doi: 10.1056/NEJMoa1409077

McMurray, J. J. V., Trueman, D., Hancock, E., Cowie, M. R., Briggs, A., Taylor, M., et al. (2018). Cost-effectiveness of sacubitril/valsartan in the treatment of heart failure with reduced ejection fraction. Heart 104 (12), 1006-1013. doi: 10.1136/ heartjnl-2016-310661

Mentz, R. J., Roessig, L., Greenberg, B. H., Sato, N., Shinagawa, K., Yeo, D., et al. (2016). Heart Failure Clinical Trials in East and Southeast Asia Understanding the Importance and Defining the Next Steps. Jacc-Heart Failure 4 (6), 419-427. doi: $10.1016 /$ j.jchf.2016.01.013

National Health Commission of the People's Republic of China (2018). China Health Statistics Yearbook 2018. Beijing: Peking Union Medical College Press.

Park, S. K., Hong, S. H., Kim, H., Kim, S., and Lee, E. K. (2019). Cost-Utility Analysis of Sacubitril/Valsartan Use Compared With Standard Care in 
Chronic Heart Failure Patients With Reduced Ejection Fraction in South Korea. Clin. Ther. 41 (6), 1066-1079. doi: 10.1016/j.clinthera.2019.04.031

Pocock, S. J., Ariti, C. A., McMurray, J. J. V., Maggioni, A., Køber, L., Squire, I. B., et al. (2012). Predicting survival in heart failure: a risk score based on 39372 patients from 30 studies. Eur. Heart J. 34 (19), 1404-1413. doi: 10.1093/eurheartj/ehs337

Ponikowski, P., Voors, A. A., Anker, S. D., Bueno, H., Cleland, J. G. F., Coats, A. J. S., et al. (2016). 2016 ESC Guidelines for the diagnosis and treatment of acute and chronic heart failure: The Task Force for the diagnosis and treatment of acute and chronic heart failure of the European Society of Cardiology (ESC). Developed with the special contribution of the Heart Failure Association (HFA) of the ESC. Eur. J. Heart Failure 18 (8), 891-975. doi: 10.1002/ejhf.592

Sandhu, A. T., Ollendorf, D. A., Chapman, R. H., Pearson, S. D., and Heidenreich, P. A. (2016). Cost-Effectiveness of Sacubitril-Valsartan in Patients With Heart Failure With Reduced Ejection Fraction. Ann. Internal Med. 165 (10), 681-68 +. doi: $10.7326 / \mathrm{m} 16-0057$

Savarese, G., and Lund, L. H. (2017). Global Public Health Burden of Heart Failure. Card Fail Rev. 3 (1), 7-11. doi: 10.15420/cfr.2016:25:2

Simpson, J., Jhund, P. S., Silva Cardoso, J., Martinez, F., Mosterd, A., Ramires, F., et al. (2015). Comparing LCZ696 With Enalapril According to Baseline Risk Using the MAGGIC and EMPHASIS-HF Risk Scores: An Analysis of Mortality and Morbidity in PARADIGM-HF. J. Am. Coll. Cardiol. 66 (19), 2059-2071. doi: 10.1016/j.jacc.2015.08.878

Tribouilloy, C., Rusinaru, D., Mahjoub, H., Souliere, V., Levy, F., Peltier, M., et al. (2008). Prognosis of heart failure with preserved ejection fraction: a 5 year prospective population-based study. Eur. Heart J. 29 (3), 339-347. doi: 10.1093/eurheartj/ehm554

van der Pol, S., Degener, F., Postma, M. J., and Vemer, P. (2017). An Economic Evaluation of Sacubitril/Valsartan for Heart Failure Patients in the Netherlands. Value Health 20 (3), 388-396. doi: 10.1016/j.jval.2016.10.015

van der Pol, S., de Jong, L. A., Vemer, P., Jansen, D., and Postma, M. J. (2019). CostEffectiveness of Sacubitril/Valsartan in Germany: An Application of the Efficiency Frontier. Value Health 22 (10), 1119-1127. doi: 10.1016/j.jval.2019.06.007

Xuan, J. W., Tao, L. B., Zhu, S. Q., Zhang, M. L., Ni, Q., Sun, Q., et al. (2017). Real World Survey of Non-direct Medical Cost and Quality of Life for Heart Failure
Patients of China. China Health Insur. (03), 61-64. doi: 10.19546/j.issn.16743830.2017.3.013

Yancy, C. W., Jessup, M., Bozkurt, B., Butler, J., Casey, D. E., Colvin, M. M., et al. (2017). 2017 ACC/AHA/HFSA Focused Update of the 2013 ACCF/AHA Guideline for the Management of Heart Failure: A Report of the American College of Cardiology/American Heart Association Task Force on Clinical Practice Guidelines and the Heart Failure Society of America. J. Am. Coll. Cardiol. 70 (6), 776-803. doi: 10.1016/j.jacc.2017.04.025

Zanfina, A., Alena, M. P., Elizabeth, H., David, T., Rola, H., Celine, D., et al. (2017). Cost-effectiveness of sacubitril/valsartan in chronic heart-failure patients with reduced ejection fraction. Swiss Med. Wkly. 147, w14533. doi: 10.4414/ smw.2107.14533

Zhang, Y. H., Zhang, J., Butler, J., Yang, X. M., Xie, P. Y., Guo, D. S., et al. (2017). Contemporary Epidemiology, Management, and Outcomes of Patients Hospitalized for Heart Failure in China: Results From the China Heart Failure (China-HF) Registry. J. Cardiac Failure 23 (12), 868-875. doi: 10.1016/j.cardfail.2017.09.014

Zueger, P. M., Kumar, V. M., Harrington, R. L., Rigoni, G. C., Atwood, A., DiDomenico, R. J., et al. (2018). Cost-Effectiveness Analysis of Sacubitril/ Valsartan for the Treatment of Heart Failure with Reduced Ejection Fraction in the United States. Pharmacotherapy 38 (5), 520-530. doi: 10.1002/phar.2108

Conflict of Interest: The authors declare that the research was conducted in the absence of any commercial or financial relationships that could be construed as a potential conflict of interest.

Copyright (c) $2020 \mathrm{Wu}$, Tian, Rong, Zhang, Chen, Guo and Zhou. This is an openaccess article distributed under the terms of the Creative Commons Attribution License (CC BY). The use, distribution or reproduction in other forums is permitted, provided the original author(s) and the copyright owner(s) are credited and that the original publication in this journal is cited, in accordance with accepted academic practice. No use, distribution or reproduction is permitted which does not comply with these terms. 